\title{
12 PREDITORES DO MOBBING NOS ENFERMEIROS EM CONTEXTO HOSPITALAR ${ }^{1}$
}

| Andrea Silva Santos Pinto²; António João Santos Nunes ${ }^{3}$

\section{RESUMO}

CONTEXTO: O mobbing é um fenómeno mundial caraterístico de ambientes de trabalho inseguros, competitivos e individualistas. Constitui um grave problema psicossocial na gestão das organizações de saúde.

OBJETIVOS: Avaliar a existência de condutas de mobbing nos enfermeiros e identificar as variáveis preditoras do mobbing nos enfermeiros num Hospital da Cova da Beira, em Portugal.

METODOLOGIA: Estudo quantitativo, descritivo, correlacional e transversal. A amostra não probabilística acidental de 218 enfermeiros. Utilizou-se uma Escala de Avaliação de Condutas de Mobbing (EACM).

RESULTADOS: Verificou-se que 92,2\% dos enfermeiros inquiridos experimentaram pelo menos uma conduta de mobbing, sendo que em média os enfermeiros sofrem 12 condutas de mobbing, com um efeito quase nulo e uma intensidade fraca.

CONCLUSÕES: As variáveis preditoras do mobbing são a satisfação profissional e os superiores valorizam o desempenho. Pode-se afirmar que quanto maior a satisfação profissional e quanto mais os superiores valorizam o desempenho menor a presença de mobbing no local de trabalho.

\section{PALAVRAS-CHAVE: Assédio, não sexual; Enfermeiros; Prevenção primária}

\section{RESUMEN}

"Predicción del mobbing en los enfermeiros en contexto hospitalario"

CONTEXTO: El mobbing es un fenómeno mundial característico de ambientes de trabajos inseguros, competitivos e individualistas. Es un grave problema psicosocial en la gestión de las organizaciones de salud.

OBJETIVOS: Evaluar la existencia de conductas de mobbing en los enfermeros e identificar las variables predictoras del mobbing en los enfermeros en el Hospital de Cova da Beira, en Portugal.

METODOLOGÍA: Estudio cuantitativo, descriptivo, correlacional y transversal. La muestra es no probabilística accidental de 218 enfermeros. Se utilizó una escala de evaluación de conductos de Mobbing (EACM).

RESULTADOS: Se verificó que el 92,2\% de los enfermeros encuestados experimentaron al menos una conducta de mobbing, siendo que en promedio los enfermeros sufren 12 conductas de mobbing, con un efecto casi nulo y una intensidad débil.

CONCLUSIONES: Las variables predictoras del mobbing son la satisfacción profesional y los superiores valoran el rendimiento. Se puede afirmar que cuanto mayor sea la satisfacción profesional y cuanto más los superiores valoran el desempeño menor la presencia de mobbing en el lugar de trabajo.

\section{DESCRIPTORES: Acoso, non sexual; Enfermeros; Prevención} primaria

\begin{abstract}
"Mobbing predictors in nurses in hospital context"

BACKGROUND: Mobbing is a worldwide phenomenon, characteristic of insecure, competitive and individualistic work environments. It is a serious psychosocial problem in the management of healthcare organizations.

AIM: Evaluate the existence of mobbing behaviors on nurses and identify the variables which allow us to predict mobbing on nurses in the Cova da Beira hospital, in Portugal.

METHODS: A quantitative research, descriptive, correlational and cross-section in nature. The sample was not probabilistic and accidental, consisting of 218 nurses. A questionnaire was used containing the Mobbing Ducts Rating Scale (EACM), validated for the Portuguese population.

RESULTS: It was found that $92.2 \%$ of nurses surveyed experienced at least one mobbing behavior, and on average nurses suffer 12 mobbing behaviors, with almost no effect and a low intensity.

CONCLUSIONS: The predictor variables of mobbing are professional satisfaction and performance valuation by superiors. We can say that the higher job satisfaction and the more superiors value performance, the lower the presence of mobbing in the workplace.
\end{abstract}

\section{KEYWORDS: Harassment, non-sexual; Nurses; Primary pre- vention}

Submetido em 30-12-2017

Aceite em 27-03-2018

\footnotetext{
1 Extraído de Dissertação de Mestrado Mobbing em Ambiente Hospitalar, 2015, Universidade da Beira Interior.

2 Mestre em Gestão de Unidades de Saúde; Título Profissional; Enfermeira no Centro Hospitalar Cova da Beira, Serviço de Especialidades Médicas, andreass.enf@gmail.com

3 Doutor em Gestão; Professor Auxiliar na Universidade da Beira Interior, Departamento de Gestão e Economia, Centro de Investigação NECE-UBI, Covilhã, anunes@ubi.pt 


\section{INTRODUÇÃO}

Vivemos numa época em que as condições de trabalho são marcadas pela insegurança e pela ambição desmedida de progressão na carreira. Estes fatores geram condutas antiéticas, condutas de assédio moral/psicológico ou mobbing para com os outros, com o objetivo de destrui-los, visto estes constituírem potenciais ameaças à concretização dos objetivos (Tekin \& Bulut, 2014; João, 2013).

O mobbing é um fenómeno antigo, porém a sua discussão mundial é recente. Em Portugal, o mobbing é caraterizado por uma ou várias condutas humilhantes, degradantes e hostis, efetuadas intencionalmente por um ou mais trabalhadores contra outro que possa ser percebido como potencial ameaça.

Estas condutas de agressão são efetuadas de forma repetida e sistemática, causando lentamente a destruição da vítima, afetando a sua saúde física e mental (João, 2013).

O mobbing é um fenómeno cuja incidência tem tendência a aumentar progressivamente e cerca de $8,1 \%$ da população ativa na Europa é vítima de mobbing (Luongo, Freitas \& Fernandes, 2011). Em Portugal existem poucos estudos sobre este fenómeno, o que torna necessário a abordagem deste fenómeno psicossocial.

Por representar um fenómeno multicausal, não é possível individualizar uma causa, como única, no processo de mobbing. Vários investigadores têm procurado identificar as variáveis determinantes para a ocorrência do mobbing, como as caraterísticas sociais e organizacionais, caraterísticas demográficas, caraterísticas pessoais da vítima, do agressor e dos espetadores. Segundo João (João, 2013) as caraterísticas do mobbing dependem de cada situação, tendo em conta o tipo de trabalho, as normas da organização, os intervenientes e a cultura organizacional. Segundo Amazarrary (2010) a satisfação com a chefia, com os colegas, comprometimento organizacional afetivo e a satisfação com a natureza do trabalho, são variáveis preditoras de mobbing. A satisfação tem relação inversa com o mobbing, ao seja quanto mais insatisfeitos estiverem os sujeitos, maior o mobbing.

Também os estudos de López-Cabarcos, Vázquez-Rodríguez \& Montes-Piñeiro (2010) corroboram com os anteriores, pois segundo estes o número de comportamentos mobbing está relacionado de forma negativa com a satisfação no trabalho.
Desta forma, a perceção do ambiente de trabalho, a satisfação com a profissão e a valorização do desempenho pelos superiores quando percecionados negativamente indiciam comportamentos favoráveis à prática de condutas hostis e desprovidas de ética (Carvalho, 2010). Este fenómeno, tem consequências a curto e a longo prazo, que podem ser devastadoras a nível individual (pode causar problemas de saúde físicos e psíquicos na vítima), organizacional, social e financeiro (Tuckey \& Neall, 2014).

Embora existam várias profissões de risco, mas os enfermeiros representam uma profissão de risco acrescido, pelas caraterísticas peculiares da profissão como o stress relacionado com: o trabalho por longos períodos, a excessiva carga de trabalho, períodos de descanso inadequados entre outros (Luongo et al., 2011; Tekin \& Bulut, 2014).

Este estudo tem como objetivos: i) avaliar a existência de condutas de mobbing nos enfermeiros num Hospital da Cova da beira, em Portugal; ii) identificar as variáveis que podem ser preditoras do mobbing nos enfermeiros num hospital da Cova da Beira, em Portugal.

\section{Hipóteses}

As variáveis satisfação profissional e valorização do desempenho são preditoras da presença de mobbing nos enfermeiros.

\section{METODOLOGIA}

De acordo com os objetivos definidos, optou-se por um estudo metodológico com as seguintes caraterísticas: investigação não experimental (Freixo, 2009). A análise é quantitativa (Prodanov \& Freitas, 2013). É um estudo descritivo (Gil, 2009; Prodanov \& Freitas, 2013); analítico e correlacional, pois descreve as caraterísticas de determinada população ou fenómeno e estabelece relações entre as variáveis em estudo (Gil, 2009). De acordo com o período e sequência, é um estudo do tipo retrospetivo e transversal, uma vez que a recolha de dados irá estabelecer um corte com o passado e o futuro (Freixo, 2009).

A população alvo do estudo foi constituída por 395 enfermeiros num hospital da Cova da Beira. A técnica de amostragem foi não probabilística, acidental. De todos os participantes recolheram-se 218 questionários totalmente preenchidos, constituindo este o número final da amostra. 
Os dados foram recolhidos através da aplicação de um questionário com a EACM (João, 2012), validada para a população portuguesa por João (2012).

O questionário é constituído por uma parte que caracteriza a amostra e, outra parte, que caracteriza o mobbing nos enfermeiros, através da Escala de Avaliação de Condutas de Mobbing (EACM).

A EACM é constituída por 55 itens, agrupados em seis dimensões, compostos de enunciados, cada um acompanhado de uma escala tipo likert com scores de 0 a 6 , onde 1 significa nunca e 6 todos os dias. As respostas obtidas, ao serem agrupadas permitem a obtenção de informações que permitem caraterizar o mobbing, denominados índices globais de mobbing (João, 2012), sendo estes: o Número Total das Estratégias de Assédio Psicológico (NEAP), o Índice Global de Assédio Psicológico (IGAP) e o Índice Médio de Assédio Psicológico (IMAP). A recolha de dados ocorreu de 6 de setembro a 7 de novembro de 2014, mediante a aplicação de um pré-teste. Terminada a fase de recolha de dados, procedeu-se ao seu tratamento estatístico, através do programa Statistical Package for Social Sciences (SPSS), versão 22.0.

$\mathrm{O}$ instrumento de recolha de dados, demonstrou uma consistência excelente ( $\alpha=0,965 ; n=55$ itens), tal como também foi obtido pela autora da escala João (2012). Os valores de alfa Cronbach obtidos (a variar entre 0,783 e 0,926 ) demonstram uma boa fiabilidade para cada uma das dimensões.

Antes de se iniciar a investigação foram seguidos os procedimentos éticos/legais, solicitada e obtida autorização ao Conselho de Administração, Conselho de Ética do Hospital em estudo (CAAE 56/2014) e obtida autorização da autora da escala.

\section{RESULTADOS E DISCUSSÃO}

Em termos sociodemográficos $73 \%$ dos enfermeiros pertencem ao género feminino. A maioria dos inquiridos possui idades entre os 25 e 59 anos, sendo a média de idades 39 anos. A grande maioria é casado $(66,1 \%)$ e tem filhos (73,9\%). Os enfermeiros estão distribuídos por vinte e oito serviços, maioritariamente exercem as suas funções nos serviços de: Bloco Operatório $(8,7 \%)$ e Urgência Geral (7,3\%). 54,6\% pertencem aos quadros da função pública e $80,3 \%$ trabalham em regime de turnos, são assíduos (98,1\%), maioritariamente exercem a profissão de enfermagem entre 2-10 anos, sendo que $73,9 \%$ possui a licenciatura.
Em relação à valorização do desempenho pelos superiores, 57,3\% afirmaram que os superiores valorizam o seu trabalho e $52,3 \%$ referem mesmo estar satisfeitos com a sua profissão. Enquanto que $82,1 \%$ dos enfermeiros mencionam que não existem circunstâncias não profissionais a afetar o seu desempenho.

Em relação à temática do mobbing, a maioria $(71,6 \%)$ refere que já ouviu falar sobre o tema, sendo que $92,2 \%$ dos respondentes experimentaram, pelo menos, uma conduta de mobbing. Em média os enfermeiros sofrem 12 condutas de mobbing no local de trabalho, com um efeito quase nulo (IGAP $=0,5)$ e uma intensidade fraca (IMAP=1,8).

$\mathrm{O}$ facto de os enfermeiros viverem condutas de intensidade fraca, não quer dizer que a longo prazo as consequências sejam as mesmas, pois, no nível individual, a duração excessiva ou a magnitude das condutas de mobbing podem conduzir a patologias graves, ou agravar problemas já existentes (Gil-Monte, 2014). Estes resultados apresentam valores sensivelmente superiores aos evidenciados nos estudos de Carvalho (2010), João (2013) e Saraiva \& Pinto (2011) em que cada enfermeiro experimenta em média 11 condutas de mobbing, com um efeito e uma intensidade reduzida.

Dos métodos utilizados pelo agressor as condutas mais experimentadas pelos enfermeiros correspondem às dimensões Bloqueio à Comunicação e ao Progresso (BCP) (média=0,7), Difamação Pessoal (DP) (média $=0,3)$ e Sobrecarga e Desprestígio Laboral (SDL) (média $=0,3$ ). Sendo na dimensão BCP as condutas mais frequentes, "algumas vezes por semana" ou "todos os dias": "quando falo interrompem o meu discurso" $(11,9 \%)$, "os meus superiores não me deixam falar ou expressar" $(3,7 \%)$ e "controlam excessivamente o meu horário" (2,8\%). Na dimensão DP as condutas sofridas com maior frequência, "algumas vezes por semana" ou "todos os dias", foram: "fazem circular rumores ou boatos falsos sobre a minha vida privada", "criticam a minha vida privada" e "gozam e/ou criticam as minhas convicções políticas e/ou religiosas", apresentando igual percentagem de $0,5 \%$. Em relação à dimensão SDL as condutas mais sofridas "algumas vezes por semana" ou "todos os dias", foram: "atribuem-me mais trabalho do que aquele que posso efetivamente realizar" e "sou forçado(a) a realizar tarefas que vão contra os meus princípios éticos", todas com iguais valores percentuais $(1,4 \%)$. 
Todas as expressões de vivência de condutas de mobbing supracitadas demonstram estratégias utilizadas pelo agressor com o objetivo de desconsiderar e destabilizar a vítima a ponto de comprometer a comunicação, limitando ou negando o direito a fazer-se ouvir, assim como, desacreditar ou desprestigiar a vítima no seu local de trabalho (João, 2012). A vítima é desvalorizada através de críticas e/ou falsos rumores sobre a sua vida pessoal, da atribuição de tarefas abusivas ou difíceis de realizar, que agem contra os seus princípios éticos. As condutas de BCP, DP e SDL representam agressões dissimuladas e não deixam provas físicas, reforçando a ideia de que o mobbing se carateriza por um conjunto de condutas de caráter psicológico.

$\mathrm{O}$ mobbing representa uma realidade presente nos profissionais de enfermagem, pois 5 em cada 100 enfermeiros inquiridos têm consciência que são vítimas de mobbing no seu local de trabalho. Contudo, mais de metade da amostra refere sofrer pelo menos uma conduta de agressão, não se assumindo como vítima. Dos enfermeiros que têm consciência de ser vítimas de mobbing, a maioria (2,3\%) sofre de mobbing desde há 6 meses e todas as vítimas tiveram necessidade de denunciar/compartilhar a situação recorrendo a apoio de familiares e colegas $(1,4 \%)$. O mobbing vivido pelos enfermeiros é principalmente do tipo descendente, pois a maior parte dos agressores é o "enfermeiro chefe ou superior hierárquico" $(1,8 \%)$. As consequências na saúde física e psíquica da vítima têm um potencial efeito devastador, destacando-se os problemas "psíquicos, comportamentais, cardíacos e respiratórios”. Estes resultados também são apoiados por outras investigações (Gil-Monte, 2014; Luongo et al., 2011; Tekin \& Bulut, 2014). No que concerne às medidas preventivas descritas pelos enfermeiros para prevenção do mobbing foram mencionadas várias estratégias, as quais foram agrupadas, em medidas direcionadas para a organização (65), para o indivíduo (41) e para a sociedade (3). Relativamente às medidas direcionadas para a organização foi sugerido com maior frequência: uma "comunicação eficaz" (15), "formação sobre a temática" e "chefias com capacidade de liderança, valores de justiça e imparcialidade" (com igual frequência 9), "gestão de conflitos e trabalho em equipa" (com igual frequência 5). No que diz respeito às medidas direcionadas para o indivíduo, foi sugerido: "respeitar e saber ouvir" (18), "denunciar a autoridades competentes" (12), "bom relacionamento do indivíduo com a equipa multidisciplinar" (11).
Nas medidas direcionadas para a sociedade os enfermeiros sugeriram a "realização de campanhas de sensibilização da sociedade em geral" (3).

Recorreu-se à estatística inferencial, verificando-se as relações formuladas na hipótese de investigação, aceitando-se ou rejeitando-se a hipótese.

Numa primeira fase analisaram-se as correlações existentes, através da Correlação de Pearson, entre as variáveis independentes (sociodemográficas, socioprofissionais e pessoais) e a variável dependente mobbing, nos três índices globais. Verificou-se a existência de correlações positivas e negativas. O objetivo foi verificar se existem outras variáveis estatisticamente significativas para além das variáveis que entram na hipótese.

As únicas variáveis estatisticamente bastante significativas, nos três índices IGAP, NEAP e IMAP são a satisfação profissional e superiores valorizam o desempenho. Constatou-se que as variáveis satisfação profissional (IGAP, NEAP e IMAP; $r=-0,241, r=-0,257$, $\mathrm{r}=-0,221$ ) e a valorização do desempenho (IGAP, NEAP e IMAP; $r=-0,301, r=-0,350, r=-0,263)$, estão negativamente correlacionadas com o mobbing nos três índices globais (IGAP, NEAP e IMAP; $\mathrm{P}=0,000$, $\mathrm{P}=0,000, \mathrm{P}=0,001$ e IGAP, NEAP e IMAP; $\mathrm{P}=0,000$, $\mathrm{P}=0,000, \mathrm{P}=0,000)$.

Assim, as variáveis a incluir nos modelos de regressão para o IGAP (Tabela 1), NEAP (Tabela 2) e IMAP (Tabela 3) foram: satisfação profissional e superiores valorizam o desempenho.

Efetuou-se a análise de regressões lineares múltiplas para testar o valor preditivo das variáveis independentes (satisfação profissional e superiores valorizam o desempenho) em relação à variável dependente (mobbing).

O método de estimação foi o "enter", o qual obriga à inclusão de todas as variáveis independentes no modelo de regressão, quer sejam ou não significativas (Pestana \& Gajeiro, 2008).

Conforme se pode visualizar na Tabela 1 o modelo de regressão ajustado é altamente significativo $(\mathrm{F}=13,173$; $\mathrm{p}=0,000)$.

De acordo com as estimativas dos parâmetros de regressão é possível constatar que as variáveis satisfação profissional $(\beta=-0,248 ; p=0,001)$ e superiores valorizam o desempenho $(\beta=-0,148 ; p=0,036)$, são variáveis preditoras da intensidade global do mobbing. 
Tabela 1 -Regressão linear múltipla entre as variáveis independentes e o IGAP

\begin{tabular}{|c|c|c|c|c|c|}
\hline \multicolumn{6}{|c|}{ Variável dependente: IGAP } \\
\hline \multicolumn{6}{|l|}{$\mathrm{R}=0,330$} \\
\hline \multicolumn{6}{|l|}{$\mathrm{R} 2=0,109$} \\
\hline \multicolumn{6}{|c|}{$\mathrm{R} 2$ ajustado $=0,101$} \\
\hline \multicolumn{6}{|c|}{ Erro padrão de estimativa $=0,477$} \\
\hline \multicolumn{6}{|c|}{ Pesos de Regressão } \\
\hline $\begin{array}{l}\text { Variáveis In- } \\
\text { dependentes }\end{array}$ & $\begin{array}{l}\text { Coefici- } \\
\text { ente } \beta\end{array}$ & $\begin{array}{l}\text { Coefi } \\
\text { Padro }\end{array}$ & $\begin{array}{l}\text { ciente } \\
\text { onizado }\end{array}$ & $t$ & $\mathbf{p}$ \\
\hline (Constante) & 0,587 & & & 10,921 & $0,000^{* * *}$ \\
\hline $\begin{array}{l}\text { Superiores } \\
\text { Valorizam o } \\
\text { Desempenho }\end{array}$ & $-0,248$ & $-0,24$ & & $-3,510$ & $0,001^{* * *}$ \\
\hline $\begin{array}{l}\text { Satisfação } \\
\text { Profissional }\end{array}$ & $-0,148$ & $-0,147$ & & $-2,115$ & $0,036^{\star}$ \\
\hline \multicolumn{6}{|c|}{ Análise de Variância } \\
\hline Efeito & $\begin{array}{l}\text { Soma } \\
\text { Quadra- } \\
\text { dos }\end{array}$ & $\overline{G L}$ & $\begin{array}{l}\text { Média } \\
\text { Quadra- } \\
\text { dos }\end{array}$ & $\mathbf{F}$ & $\mathbf{p}$ \\
\hline Regressão & 5,992 & 2 & 2,996 & \multirow[t]{3}{*}{13,173} & \multirow[t]{3}{*}{$0,000^{\star \star \star}$} \\
\hline Resíduos & 48,898 & 215 & 0,227 & & \\
\hline Total & 54,890 & 217 & & & \\
\hline
\end{tabular}

${ }_{* * *}<<0,001$ - diferença estatística altamente significativa

Como se pode analisar no Tabela 2 o modelo de regressão ajustado para o NEAP é altamente significativo $(\mathrm{F}=17,576 ; \mathrm{p}=0,000)$. Em conformidade com as estimativas dos parâmetros de regressão é possível constatar que as variáveis superiores valorizam o desempenho $(\beta=-5,779 ; p=0,000)$ e satisfação profissional $(\beta=-2,792 ; p=0,036)$, são estatisticamente significativas, logo constituem-se como variáveis preditoras do número de condutas do mobbing. Verifica-se que quanto mais os indivíduos estiverem profissionalmente satisfeitos e quanto mais os superiores valorizam o desempenho menor é o número de condutas do mobbing.
Tabela 2 -Regressão linear múltipla entre as variáveis independentes e o NEAP (formatar título e centrar tabela)

\begin{tabular}{|c|c|c|c|c|c|}
\hline \multicolumn{6}{|c|}{ Variável dependente: NEAP } \\
\hline \multicolumn{6}{|l|}{$\mathrm{R}=0,375$} \\
\hline \multicolumn{6}{|l|}{$\mathrm{R} 2=0,141$} \\
\hline \multicolumn{6}{|c|}{ R2 ajustado $=0,133$} \\
\hline \multicolumn{6}{|c|}{ Erro padrão de estimativa $=9,037$} \\
\hline \multicolumn{6}{|c|}{ Pesos de Regressão } \\
\hline $\begin{array}{l}\text { Variáveis In- } \\
\text { dependentes }\end{array}$ & $\begin{array}{l}\text { Coefici- } \\
\text { ente } \beta\end{array}$ & $\begin{array}{l}\text { Coef } \\
\text { Padr }\end{array}$ & $\begin{array}{l}\text { ciente } \\
\text { nizado }\end{array}$ & $t$ & $\bar{p}$ \\
\hline (Constante) & 16,292 & & & 15,993 & $0,000^{* * *}$ \\
\hline $\begin{array}{l}\text { Superiores } \\
\text { Valorizam o } \\
\text { Desempenho }\end{array}$ & $-5,779$ & $-0,29$ & & $-4,314$ & $0,000^{* \star *}$ \\
\hline $\begin{array}{l}\text { Satisfação } \\
\text { Profissional }\end{array}$ & $-2,792$ & $-0,14$ & & $-2,105$ & $0,036^{*}$ \\
\hline \multicolumn{6}{|c|}{ Análise de Variância } \\
\hline Efeito & \begin{tabular}{|l|} 
Soma \\
Quadra- \\
dos \\
\end{tabular} & GL & $\begin{array}{l}\text { Média } \\
\text { Quadra- } \\
\text { dos }\end{array}$ & $F$ & $\mathbf{p}$ \\
\hline Regressão & 2870,935 & 2 & 1435,467 & \multirow[t]{3}{*}{17,576} & \multirow[t]{3}{*}{$0,000^{\star * x}$} \\
\hline Resíduos & 17559,492 & 215 & 81,672 & & \\
\hline Total & 20430,427 & 217 & & & \\
\hline
\end{tabular}

Na Tabela 3 encontra-se o modelo de regressão ajustado para o IMAP, sendo altamente significativo $(\mathrm{F}=9,556 ; \mathrm{p}=0,000)$, apesar de apresentar uma qualidade fraca $(\mathrm{r} 2=0,086)$. De acordo com as estimativas dos parâmetros de regressão é possível constatar-se que as variáveis superiores valorizam o desempenho $(\beta=-0,257 ; p=0,004)$ e satisfação profissional $(\beta=-$ $0,173 ; p=0,052)$ são variáveis preditoras da intensidade média do mobbing.

Deste modo, quanto mais os indivíduos estiverem profissionalmente satisfeitos e quanto mais os superiores valorizam o desempenho, menor é a intensidade média do mobbing. 
Tabela 3 -Regressão linear múltipla entre as variáveis independentes e o IMAP Formatar título e centrar tabela)

\begin{tabular}{|c|c|c|c|c|c|}
\hline \multicolumn{6}{|c|}{ Variável dependente: IMAP } \\
\hline \multicolumn{6}{|l|}{$\mathrm{R}=0,294$} \\
\hline \multicolumn{6}{|l|}{$\mathrm{R} 2=0,086$} \\
\hline \multicolumn{6}{|c|}{$\mathrm{R} 2$ ajustado $=0,077$} \\
\hline \multicolumn{6}{|c|}{ Erro padrão de estimativa $=0,58745$} \\
\hline \multicolumn{6}{|c|}{ Pesos de Regressão } \\
\hline $\begin{array}{l}\text { Variáveis In- } \\
\text { dependentes }\end{array}$ & $\begin{array}{l}\text { Coefici- } \\
\text { ente } \beta\end{array}$ & $\begin{array}{l}\text { Coef } \\
\text { Padr }\end{array}$ & $\begin{array}{l}\text { ciente } \\
\text { onizado }\end{array}$ & $\mathbf{t}$ & $\mathbf{p}$ \\
\hline (Constante) & 1,793 & & & 26,765 & $0,000^{* * *}$ \\
\hline $\begin{array}{l}\text { Superiores } \\
\text { Valorizam o } \\
\text { Desempenho }\end{array}$ & $-0,257$ & $-0,20$ & & $-2,881$ & $0,004^{\star *}$ \\
\hline $\begin{array}{l}\text { Satisfação } \\
\text { Profissional }\end{array}$ & $-0,173$ & $-0,14$ & & $-1,955$ & $0,052^{*}$ \\
\hline \multicolumn{6}{|c|}{ Análise de Variância } \\
\hline Efeito & $\begin{array}{l}\text { Soma } \\
\text { Quadra- } \\
\text { dos }\end{array}$ & GL & $\begin{array}{l}\text { Média } \\
\text { Quadra- } \\
\text { dos }\end{array}$ & $\mathbf{F}$ & $\mathbf{p}$ \\
\hline Regressão & 6,596 & 2 & 3,298 & \multirow[t]{3}{*}{9,556} & \multirow[t]{3}{*}{$0,000^{\star * *}$} \\
\hline Resíduos & 69,710 & 202 & 0,345 & & \\
\hline Total & 76,306 & 204 & & & \\
\hline
\end{tabular}

Assim, após análise dos três modelos de regressão, aceita-se a hipótese formulada para as variáveis em estudo.

Pode dizer-se que as variáveis independentes preditoras de mobbing nomeadamentea satisfação profissional e a valorização do desempenho pelos superiores hierárquicos, apresentam uma relação inversa com o mobbing.

Verifica-se que, quanto maior a satisfação profissional e quanto mais os superiores valorizam o desempenho, menor será a presença de mobbing.

Estes resultados estão em consonância com os estudos de López-Cabarcos et al. (2010), pois a satisfação profissional é uma variável preditora do mobbing. A satisfação assume uma relação inversa com o mobbing, ou seja, quanto mais insatisfeitos estiverem os sujeitos, maior será o mobbing. Também o estudo de Carvalho (2010) é semelhante, pois a perceção do ambiente de trabalho, a satisfação com a profissão e a valorização pelos superiores, quando percecionados negativamente, indiciam comportamentos favoráveis à prática de condutas de mobbing.

\section{IMPLICAÇÕES PARA A PRÁTICA CLÍNICA}

Uma vez que os sintomas do mobbing evoluem a nível clínico, é importante o diagnóstico precoce, para uma prevenção e intervenção atempada. Segundo a Comissão para a Igualdade no Trabalho e no Emprego - CITE (2013) a avaliação pode ser feita através dos serviços de Higiene e Segurança no Trabalho internos ou externos. Estes serviços devem ter consultas de avaliação de riscos psicossociais, e uma equipa multidisciplinar com enfermeiro, médico e psicólogo. É papel destes profissionais trabalhar articuladamente de forma a prevenir, diagnosticar e minimizar os efeitos do mobbing no trabalhador. Também podem existir serviços de psicologia internos (Gil-Monte, 2014). Podendo em casos extremos ser necessário um acompanhamento por parte de um psiquiatra.

Os estudos realizados por Moroni \& Dabos (2014) mencionam que as organizações podem influenciar positivamente o ambiente laboral vivenciado pelos trabalhadores por melhorar a comunicação interna, fomentar uma cultura de transparência e prestação de contas, selecionando e treinando líderes capazes de fazerem uma gestão correta de emoções e de conflitos. Neste contexto, o psicólogo pode intervir através de formação e capacitação destes agentes, através do desenvolvimento de programas de coaching (Saam, 2010).

\section{CONCLUSÃO}

O mobbing envolve condutas destruidoras, agressivas, violentas, manipuladoras e de perseguição por parte do agressor. Estas ocorrem de maneira intencional e frequente no trabalho por um ou mais agressores, com o objetivo de destruírem e excluírem socialmente a vítima.

A caraterização da amostra refere que 92,2\% já experimentaram pelo menos uma conduta de mobbing. Verificando-se que em média os enfermeiros sofrem 12 condutas de mobbing no local de trabalho, com um efeito quase nulo e uma intensidade fraca. Dos métodos utilizados pelo agressor as condutas mais experimentadas pelos enfermeiros correspondem às dimensões BCP (média=0,7), DP (média $=0,3$ ) e SDL (média $=0,3$ ). Através do teste da hipótese concluiu-se que o mobbing está relacionado com a satisfação profissional e com o facto dos superiores valorizarem o desempenho dos enfermeiros. 
Os resultados demonstram que a satisfação profissional e os superiores valorizam o desempenho relacionamse inversamente com a presença de mobbing, ou seja, quanto maior a satisfação profissional e quanto mais os superiores hierárquicos valorizarem o desempenho profissional dos trabalhadores menor será a presença de mobbing.

Por tudo o referido, torna-se prioritário a organização investir na prevenção do mobbing, sendo que os enfermeiros inquiridos propõem algumas medidas para prevenção do mobbing aos níveis: organizacional, pessoal e social.

A defesa da dignidade humana exige a erradicação de toda e qualquer prática de mobbing nos locais de trabalho, as organizações, começando pelas chefias devem sinalizar a sua forma de atuação através da punição dos agressores e do apoio aos verdadeiramente agredidos. Cada sociedade deve enquadrar inequivocamente no seu normativo jurídico a punição do agressor, só dessa forma se podem garantir os direitos de plena cidadania aos trabalhadores que possuem no seu trabalho uma forma de satisfação profissional e de realização pessoal.

Como limitações deste estudo realça-se a utilização do método de amostragem não probabilístico, apesar de ser fácil e rápido, os resultados apenas dizem respeito à amostra utilizada, não podendo ser extrapolados com confiança para a restante população.

\section{REFERÊNCIAS BIBLIOGRÁFICAS}

Amazarray, R. M. (2010). Violência psicológica e assédio moral no trabalho enquanto expressões de estratégias de gestão. (Tese de Doutoramento não publicada). Universidade Federal do Rio Grande do Sul, Brasil.

Carvalho, G. (2010). Mobbing: assédio moral em contexto de enfermagem. Revista de Enfermagem Referência, 21, 28-42. Disponível em: https://bibliotecadigital.ipb.pt/bitstream/10198/5327/1/Mobbing\%20 Ass\%C3\%A9dio\%20Moral\%202010.PDF

Comissão para a Igualdade no Trabalho e no Emprego. (2013). Prevenção e combate de situações de assédio no local de trabalho: um instrumento de apoio à autorregulação. Disponível em: http://cite.gov.pt/pt/destaques/noticia225.html
Freixo, M. (2009). Metodologia científica - fundamentos, métodos e técnicas. Lisboa: Instituto Piaget, Epistemologia e Sociedade.

Gil, A. C. (2009). Métodos e técnicas de pesquisa social. 5. ${ }^{\mathrm{a}}$ Ed. São Paulo: Editora Atlas.

Gil-Monte, P. R. (2014). Assédio moral no trabalho. In: S. P. Gonçalves (Eds.), Psicossociologia do trabalho e das organizações, princípios e práticas. Lisboa: Ed. Pactor.

João, A. L. (2012). Relatório de mestrado em sociopsicologia da saúde. (Relatório de Mestrado não publicado). Instituto Miguel Torga, Portugal.

João, A. L. (2013). Mobbing/agressão psicológica na profissão de enfermagem. Loures: Lusociência.

López-Cabarcos, M. A.; Vázquez-Rodríguez, P. M. \& Montes-Piñeiro C. (2010). Bullying at work: Psychological antecedents and consequences on job satisfaction. Revista Latinoamericana de Psicologia, 42 (2), 214-224. Disponível em: http://www. scielo.org.co/scielo.php?script $=$ sci_arttext\&pid $=$ S0120-05342010000200005

Luongo, J.; Freitas, G. F. \& Fernandes, M. F. P. (2011). Caraterização do assédio moral nas relações de trabalho: uma revisão da literatura. Cultura de los Cuidados, 15 (30), 71-78. Disponível em: https://rua.ua.es/ dspace/bitstream/10045/18698/1/cultura_cuidados_30_10.pdf

Moroni, L. \& Dabos, G. E. (2014). Comportamientos abusivos de baja intensidad en las organizaciones: una revisión de la literatura y de sus implicâncias. Estudios Gerenciales, 30, 384-396. Disponível em: https://www.sciencedirect.com/science/article/pii/ S012359231400134X

Pestana, M. H. \& Gajeiro, J. N. (2008). Análise de dados para as ciências sociais, a complementaridade do SPSS. 5. ${ }^{a}$ Ed. Lisboa: Edições Silabo.

Prodanov, C. C. \& Freitas, E. C. (2013). Metodologia do trabalho científico, métodos e técnicas da pesquisa e do trabalho académico. 2. ${ }^{\text {a }}$ E. Rio Grande Sul: Feevale. 
Saam, N. J. (2010). Interventions in workplace bullying: A multilevel approach. European Journal of Work and Organizational Psychology, 19 (1), 51-75. Disponível em: https://www.tandfonline.com/doi/ abs/10.1080/13594320802651403

Saraiva, D. M. R. F. \& Pinto, A. S. S. (2011). Mobbing em contexto de enfermagem. Revista de Enfermagem Referência, 3(5), 83-93. Disponível em: http:// www.scielo.mec.pt/scielo.php?script=sci_arttext\&pid $=$ S0874-02832011000300009
Tekin, Y. E \& Bulut, H. (2014). Verbal, physical and sexual abuse status against operating room nurses in Turkey. Sexuality Disability, 32 (1), 85-97. Disponível em: https://link.springer.com/article/10.1007/s11195014-9339-7

Tuckey, M. R. \& Neall, A. M. (2014). A methodological review of research on the antecedents and consequences of workplace harassment. Journal of Occupational and Organizational Psychology, 87 (2), 225-257. Disponível em: https://onlinelibrary.wiley.com/doi/ abs/10.1111/joop.12059

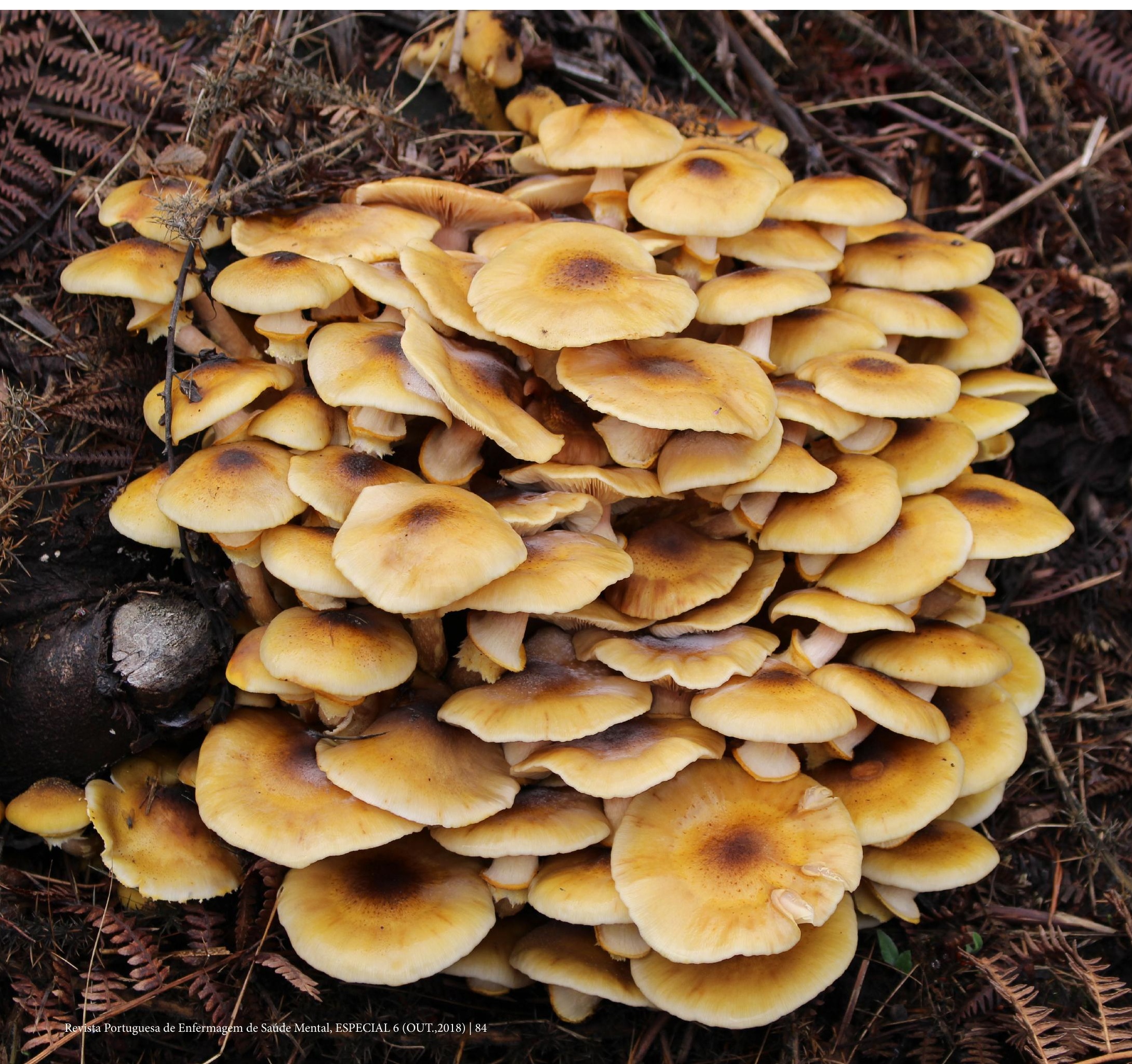

